\title{
Surgical Technique and Results of Double-door Laminoplasty at the Cervical Spine (Kurokawa's Method)-Focusing on the Change of Sagittal Alignment \\ Shigeru Hirabayashi \\ Department of Orthopaedic Surgery, Teikyo University Hospital, Tokyo, Japan
}

\begin{abstract}
Purpose: Double-door laminoplasty (DDL) at the cervical spine (Kurokawa's method) has a feature of central-splitting of the spinous processes and laminae with preserving their lengths. This procedure is technically demanding, and even now there remain some issues to be mentioned. The purpose of this study was to present the recent surgical methods and the results of DDL focusing on the changes of sagittal alignment of the cervical spine.

Methods: The sagittal alignment was examined using C2-C7 angle on X-ray films in 37 patients (33 males and 4 females, the age from 34 to 87 years old with 64.1 in average) followed up more than 1 year. Results: Preoperatively, 33 patients had lordotic alignment with an average of 14.0 degrees. Postoperatively, the alignment changed toward less lordotic or kyphotic of about 10 degrees in $67 \%$ of patients. In 3 patients among 4 with preoperative kyphosis, the change toward more kyphotic was also about 10 degrees.

Conclusions: There are three essential procedures in DDL: First, to decompress the cervical spinal cord by central splitting of the spinous processes and laminae while preserving their lengths, resulting in a symmetrical widening of the spinal canal space. Second, to maintain the widened space stably by fixing with spacers of hydroxyapatite whose configuration is almost the same as that of the widened space. Third, to suture the semispinalis muscles to the ipsilateral inferior oblique occipital muscles before closure to restore their strength to the extent possible. After DDL, the sagittal alignment of the cervical spine can be expected to be well maintained postoperatively.
\end{abstract}

\section{Introduction}

In 1973, the idea of cervical laminoplasty was first proposed by Oyama and co-workers [1] by the name of "Expansive lamina-Zplasty", where decorticated laminae are opened in a zigzag pattern, resulting in obtaining a widened spinal canal. Thereafter, various methods of cervical laminoplasty have been developed in Japan and these have gradually become common around the world.

At present, the surgical methods of cervical laminoplasty are broadly divided into two types from the viewpoint of the site of osteotomy: double-door type laminoplasty (DDL) [2-4] and opendoor type laminoplasty (ODL) [5-7]. DDL was first developed by Kurokawa in the late 1970's and reported in 1982 [2]. Thereafter, it has been modified gradually by adding various procedures in response to occurrence of various problems $[8,9]$. Among them, how to maintain the cervical alignment has been an important issue because the posterior muscles are somewhat damaged during procedure via posterior approach. One of the essential procedures of DDL is to split the spinous processes and laminae centrally while preserving their lengths to maintain the strength of posterior muscles as much as possible with ventral support. However, even now, the procedure of central splitting of the spinous processes is technically demanding. There remain some issues to be mentioned and discussed.

The purpose of this paper is to present the recent DDL surgical methods and results focusing on the changes of sagittal alignment of the cervical spine.

\section{Surgical Method}

In most patients, this surgery is performed at the levels between C3 (the third cervical) and C6, because the spinal cord is most frequently

\section{Publication History:}

Received: April 15, 2017

Accepted: May 15, 2017

Published: May 17, 2017

\section{Keywords:}

Artificial spacer, Cervical Spine, Double-door laminoplasty, Sagittal alignment, Semispinalis muscle compressed at these levels except in some patients whose enlarged ossification of the posterior longitudinal ligament (OPLL) extends toward the $\mathrm{C} 1$ and $\mathrm{C} 2$ levels [10-13].

Under general anesthesia, a Mayfield three point fixator is fixed to the cranium of a patient and connected to the operation table while maintaining the cervical spine at a slightly flexed position. Care must be taken not to hyperextend the cervical spine at the time of connection. Before central longitudinal skin incision, the level of $\mathrm{C} 2$ and $\mathrm{C} 7$ spinous processes is identified by palpating the surface of skin. The nuchal ligament is centrally incised so as not to damage the posterior branch of the posterior ramus of the cervical nerve, and the nuchal ligament is detached from the C6 and /or C7 spinous processes. By exact central advancing deep into the row of the spinous processes, the volume of bleeding can be minimized. After detaching the semispinalis muscles from the $\mathrm{C} 2$ spinous process, these muscles are marked by non-soluble threads. By detaching the paravertebral muscles from each spinous process, the laminae from the caudal side of $\mathrm{C} 2$ to the cranial side of $\mathrm{C} 7$ are exposed. The lateral sides are exposed up to the central portion of each facet joint from the $\mathrm{C} 3$ to C6 levels.

"Corresponding Author: Dr. Shigeru Hirabayashi, Department of Orthopaedic Surgery, Teikyo University Hospital, 11-1, Kaga 2-Chome, Itabashi, Tokyo, Japan; E-mail: shirab@siren.ocn.ne.jp

Citation: Hirabayashi S (2017) Surgical Technique and Results of Doubledoor Laminoplasty at the Cervical Spine (Kurokawa's Method)-Focusing on the Change of Sagittal Alignment. Int J Surg Surgical Porced 2: 118. doi: https://doi. org/10.15344/2456-4443/2017/118

Copyright: (c) 2017 Hirabayashi. This is an open-access article distributed under the terms of the Creative Commons Attribution License, which permits unrestricted use, distribution, and reproduction in any medium, provided the original author and source are credited. 
Citation: Hirabayashi S (2017) Surgical Technique and Results of Double-door Laminoplasty at the Cervical Spine (Kurokawa's Method)-Focusing on the Change of Sagittal Alignment. Int J Surg Surgical Porced 2: 118. doi: https://doi.org/10.15344/2456-4443/2017/118

Page 2 of 5

After resecting the interspinalis muscles from $\mathrm{C} 2$ to $\mathrm{C} 7$ levels, the caudal border of the $\mathrm{C} 2$ lamina is resected as a dome shape using an air drill so deep as to expose the yellow ligament at the $\mathrm{C} 2 / \mathrm{C} 3$ level. From this procedure on, the spinous processes and laminae are split centrally in order from the C3 to C6 levels. At first, a pyramidal- shaped dome osteotomy is made at the cranial base of the C3 spinous process by using a diamond drill burr of $3 \mathrm{~mm}$ in diameter to obtain a good visual field. Next, the remaining part of the spinous process is split centrally from its surface by using a diamond drill burr of $2 \mathrm{~mm}$ in diameter, and the split portion is connected to the pyramidal- shaped dome (Figure 1).These procedures enable easy performance of osteotomy of the inner cortex of the lamina, which is located deeply and hard to visualize.

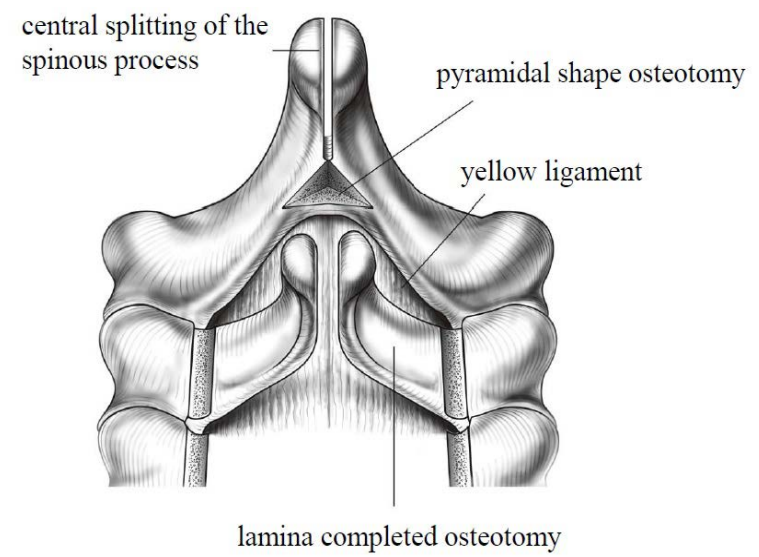

Figure 1: Pyramidal-shaped dome osteotomy at the cranial base of the spinous process and central splitting of the spinous process and lamina (partially modified [13], permitted by MIWA-SHOTEN Ltd.)

At first, a pyramidal-shaped dome osteotomy is made at the cranial base of the spinous process to obtain a good visual field. Next, the remaining part of the spinous process is split centrally from its surface and the split portion is connected to the pyramidal-shaped dome.

It is recommended that at the time of osteotomy of the inner cortex, a surgeon with right dexterity stands at the right side of the patient. To obtain well illuminated surgical field at the inner cortex, lighting should be set longitudinally from the cranial side. At each vertebra, osteotomy is initially performed at the caudal side and gradually proceeds to the cranial side, because the caudal side is slightly further away from the dura mater and the osteotomy can be made more safely. The surgeon must pay attention to the changes in color of the osteotomy site from red of cancellous bone, to white of the inner cortex, and finally to yellow of the yellow ligament and extradural fat tissue. And the changes in sound and tactile sensation delivered from the air-drill at the time of complete osteotomy of the inner cortex of lamina must also be attended. By moving an air-drill slowly, tactile sensation can become more acute. The progress of osteotomy must be checked often by lightly touching the surgical field with a probe.

After central splitting is completed at each vertebra, a longitudinal groove of $3 \mathrm{~mm}$ in width is made bilaterally at the lamina-facet junction line by resecting the outer cortex and a portion of cancellous bone (Figure 2). Care must be taken not to resect the inner cortex too deep to avoid sinking of the lamina causing radiculopathy. It is recommended that the surgeon stands at the cranial side of the patient to make the bilateral groove symmetrically. After a longitudinal groove has been made bilaterally at the C3 level, a scissors is inserted between the split spinous processes and opened to widen the space. During the time of widening, a sensation of slight resistance is adequate. The same procedures are performed up to the C6 level, and the cranial side of the C7 lamina is resected as a dome shape.

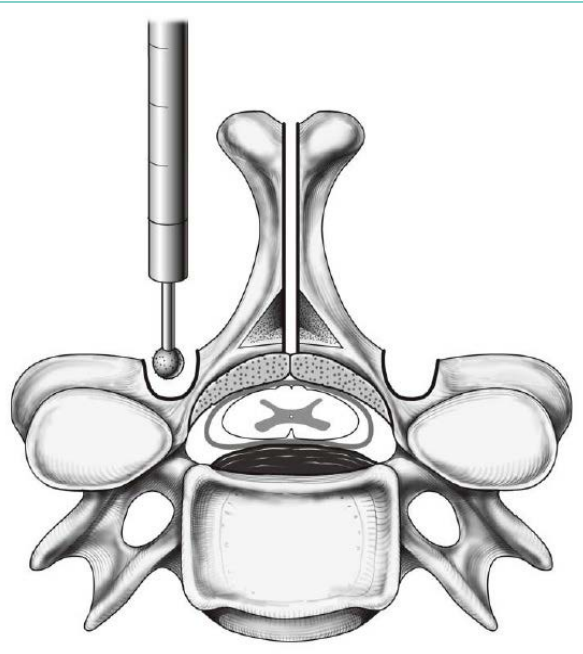

Figure 2: Formation of longitudinal groove (partially modified [13], permitted by MIWA-SHOTEN Ltd.)

A longitudinal groove of $3 \mathrm{~mm}$ in width is made bilaterally at the lamina-facet junction line by resecting the outer cortex and a part of cancellous bone.

After completing osteotomy, the constricting fibrous band above the dura mater and the hypertrophied yellow ligament are resected using a rongeur in order from the C6 to C3 levels (Figure 3). To equalize the degree of resistance of bilateral split spinous process, the depth of the longitudinal groove is adjusted by adding depth to the resection of the inner cortex. At each level, the shape of the widened space is trapezoidal, both on the axial and frontal sections, because the cranial side of the widened space is more widely opened than the caudal side. A trial spacer is inserted into the widened space and an appropriate size of actual artificial spacer (STSS spacer: HOYA CORPORATION, Tokyo, Japan) is selected. A hole of $2 \mathrm{~mm}$ in diameter to accommodate a thread for fixing the spacer is made at about $8 \mathrm{~mm}$ or more superficial from the inner plate of the lamina. The STSS spacer whose shape is the same as that of the widened space is firmly stabilized by slightly rotating to the appropriate position $[9,11-15]$. Each spacer is fixed using one or two non-soluble threads (Figure 4). In our standard method, an STSS spacer of $19 \mathrm{~mm}$ in cranial transverse length is fixed at the C5 and C6 levels (Figure 5). Before closure, the semispinalis muscles which are once detached from the $\mathrm{C} 2$ spinous process are re-sutured to the ipsilateral inferior oblique occipital muscles to restore their strength to the extent possible. After one suction drainage is set, sutures are made in each layer.

Immediately after surgery, muscle exercises of the extremities begin during bed rest. After a few days, moving around using a walker or wheelchair is allowed wearing a soft neck collar. At the end of 1 month, the collar is discarded.

\section{Methods}

The postoperative change of sagittal alignment of the cervical spine was investigated in 37 patients ( 33 males and 4 females, the age from 34 to 87 years old with 64.1 in average) followed up more than 1 year. 

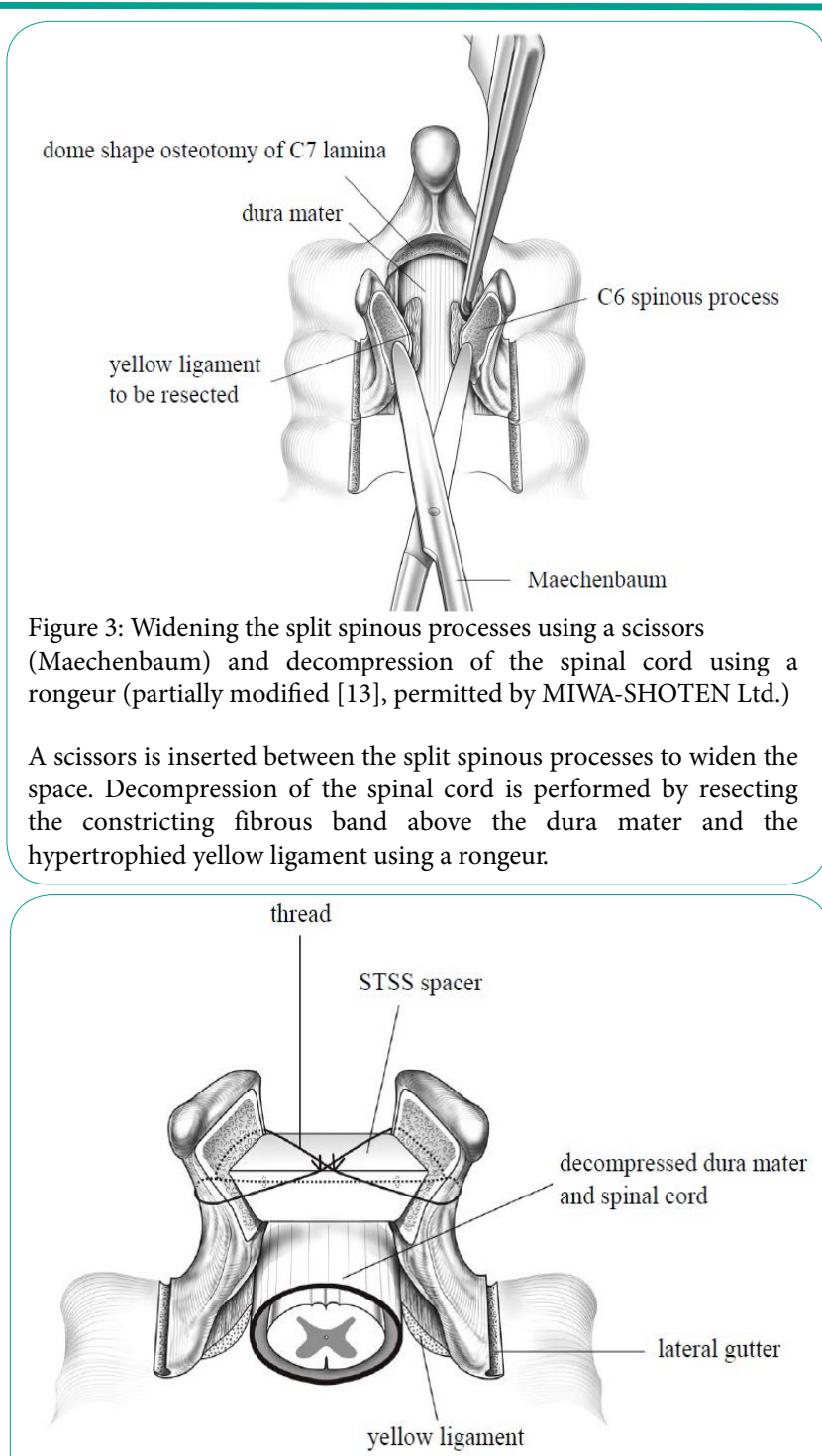

Figure 4: Fixation of the STSS spacer seen from the cranial side (partially modified [13], permitted by MIWA-SHOTEN Ltd.)

Each spacer is fixed using one or two non-soluble threads. It is noted that the cranial side is more widely opened than the caudal side.

The operated level was between C3 and C6 or C7. The number of operated vertebra was 5 in 12 patients and 4 in 25 patients. The sagittal alignment was measured using C2-C7 angle on X-ray films.

\section{Results}

In 33 of 37 patients, the preoperative alignment was lordotic with an average of $14.0 \pm 7.0$ (range 0.1-29.9) degrees. Postoperatively, in 16 patients, alignment became less lordotic with an average change of $8.3 \pm 4.6$ (1.1-20.2) degrees. In 6 patients, the alignment changed to kyphotic with an average change of $11.7 \pm 4.5$ (6.9-17.5) degrees. In 11 patients, the alignment became more lordotic with an average change of 5.6 $\pm 4.0(0.3-19.5)$ degrees. In contrast, in the other 4 patients, the preoperative alignment was kyphotic with an average of $10.7 \pm 7.3$ (3.0-18.7) degrees. Postoperatively, in 3 of these patients, alignment became more kyphotic with an average change of $12.8 \pm 4.5$ (7.9-19.6) degrees. In the remaining one patient, it changed to less kyphotic by 7.1 degrees (Figure 6).

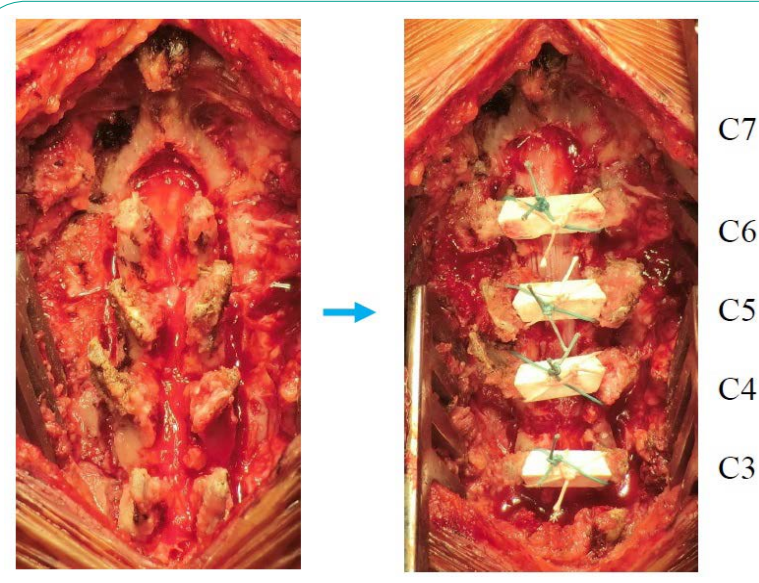

(view from the cranial side)

Figure 5: Photos during surgery seen from the cranial side. Complete decompression of the spinal cord (left) and fixation of the spacers (right).

In summary, 33 of 37 patients had lordotic alignment with an average of 14.0 degrees preoperatively. This changed toward less lordotic or kyphotic postoperatively by about 10 degrees in $67 \%$ of patients. In 3 of 4 patients with preoperative kyphosis, the change toward more kyphotic was also by about 10 degrees.

Simple X-ray films (Figure 7), CT (Figure 8) and MRI (Figure 9) are presented of a 46-year-old male with cervical spondylotic myelopathy who underwent DDL as a representative case.

\section{Discussion}

The essential procedures of DDL are as follows: 1) To decompress the cervical spinal cord by central splitting of the spinous processes and laminae while preserving their lengths, resulting in a symmetrical widening of the spinal canal space [16-19]. 2) To maintain the widened space stably by fixing with spacers of hydroxyapatite whose configuration is almost the same as that of the widened space $[9,11$ 15]. 3) To suture the semispinalis muscles to the ipsilateral inferior oblique occipital muscles before closure to restore their strength to the extent possible [8].

In this study, whether the preoperative alignment was lordotic or kyphotic, the postoperative change was of about 10 degrees. The sagittal alignment of the cervical spine was revealed to be well maintained postoperatively after DDL. It can be thought that the procedures of preserving the length of spinous processes to maintain the strength of posterior muscles as much as possible by ventrally supporting and re-suturing the semispinalis muscles to ipsilateral inferior oblique occipital muscles were effective.

In our series, the relationship between the kyphosis after laminoplasty and postoperative muscular atrophy of the paravertebral muscles of the cervical spine has not been investigated and therefore unknown. In general, postoperative muscular atrophy of the paravertebral muscles occurs more or less after cervical surgeries via posterior approach [20] because the posterior muscles are somewhat damaged during procedure of detaching from bones, stretching, and compressing. On MRI in this study, muscular atrophy can certainly be seen to some extent. However, this change is likely caused by not kyphosis but detaching the muscles from bones because the postoperative alignment change of the cervical spine was at most 10 degrees. 
Citation: Hirabayashi S (2017) Surgical Technique and Results of Double-door Laminoplasty at the Cervical Spine (Kurokawa's Method)-Focusing on the Change of Sagittal Alignment. Int J Surg Surgical Porced 2: 118. doi: https://doi.org/10.15344/2456-4443/2017/118
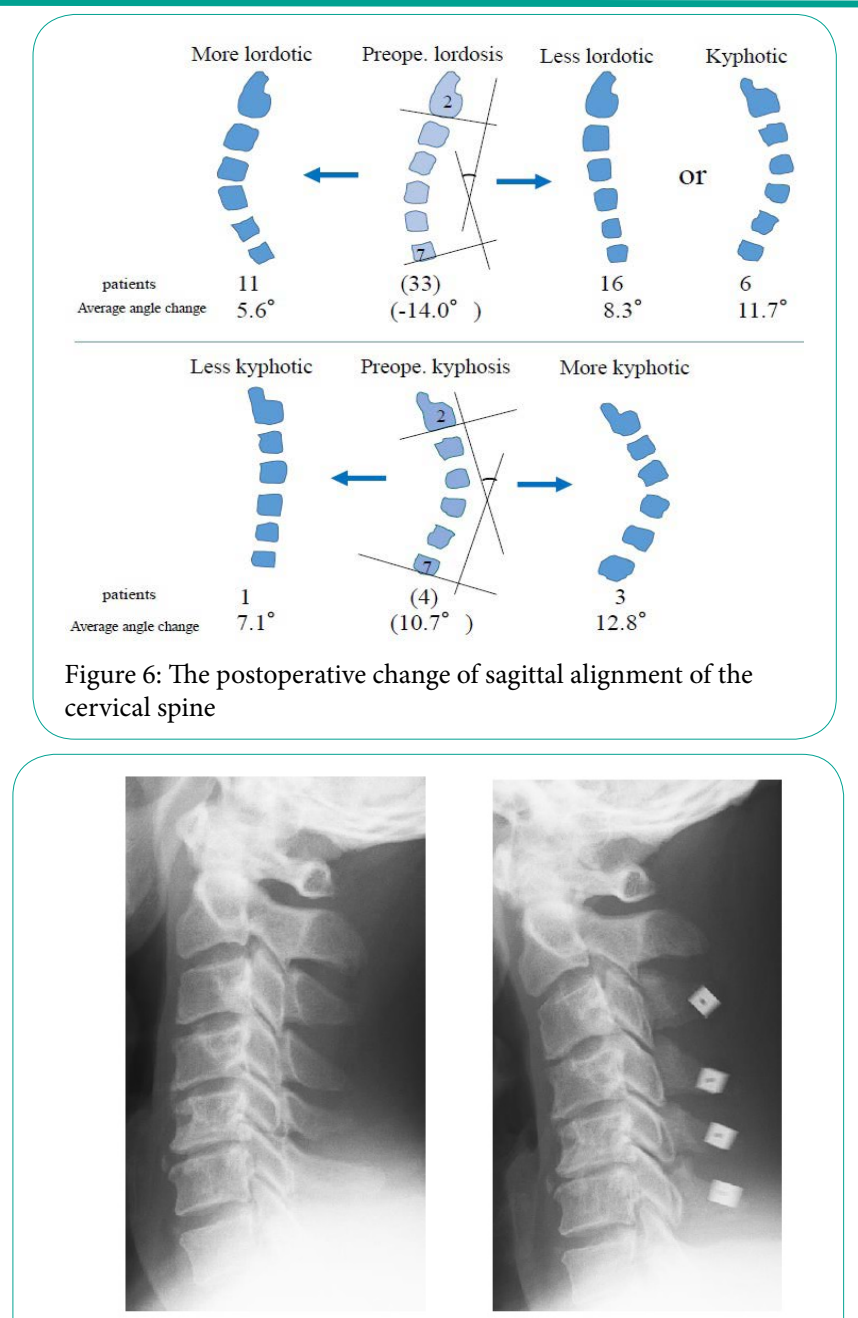

Figure 7: Simple X-ray films of a 46-year-old male with cervical spondylotic myelopathy. Before surgery (left)and after surgery (right)

CT
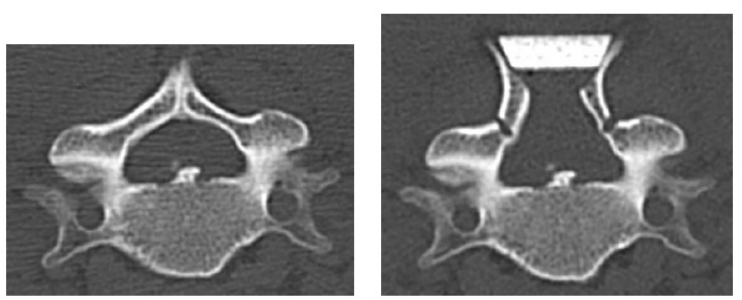

C6

Figure 8: CT at C6 level. Before surgery (left)and after surgery (right) A wide spinal canal was obtained after surgery.

As reported previously $[17,18]$, the expansion ratio of the spinal canal was about $150 \%$ at the C5 level, and about $140 \%$ at the C6 level when a spacer of $19 \mathrm{~mm}$ in cranial transverse diameter was fixed at $8 \mathrm{~mm}$ superficial from the inner cortex of the lamina.

Although postoperative C5 palsy has not been encountered in our series, the incidence in double-door laminoplasty was reported to be $4.3 \%$ [21]. At present, concerning the causes of C5 palsy, there are two main theories: nerve root injury and segmental spinal cord injury. But

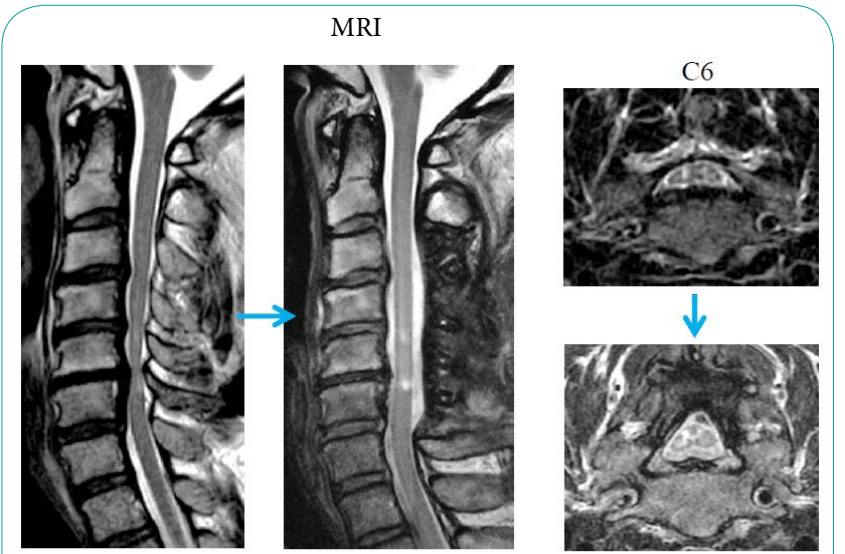

Figure 9: Sagittal and axial sections (C6 level) of MRI. Before surgery and after surgery.

The spinal cord was completely decompressed after surgery.

it is unclear yet which of these is correct. As reported previously [19], based on our anatomical analysis using cadavers, we have concluded that this palsy is most likely caused by the $\mathrm{C} 5$ nerve root compression and stretch near the exit of the foramen. The essences of our analysis are as follows: 1) Among the cervical nerve roots composed of the brachial plexus, the distance between the division from the dura mater and the exit of the foramen is shortest at the C5 nerve root. This means that the capacity of moving freely is estimated to be smallest at the C5 nerve root, 2) Anterior rootlet runs adjacent to the narrowest part of the foramen, that is, the tip of the superior facet joint, 3) Medial branch of the posterior ramus runs in the shortest distance in contact with the lateral side of the facet joint column. If the multifidus muscles are severely retracted laterally by hooks during posterior surgery, not only the medial branch of the posterior ramus but also the anterior and posterior rami and the anterior rootlet are simultaneously stretched and compressed against adjacent bone structures. In patients with hypertrophied facet joint due to degenerative changes, the influence of these stretches and compressions are estimated to become larger, especially at $\mathrm{C} 4$ and $\mathrm{C} 5$ levels where the incidence of anterior prominence of facet joint is highest [22]. To prevent and decrease the postoperative C5 palsy, it is recommended that too severe lateral stretch of the multifidus muscles for a long time must be avoided. During laminoplasty, intermittent relaxation of tension of the hooks to the muscles may be one method of solution.

DDL is indicated for patients with spinal canal stenosis at multiple levels or anterior space occupying lesion at two or more levels such as cervical spondylotic myelopathy, OPLL, cervical disc herniation, and destructive spondyloarthropathy due to renal disease[11, 2326]. However, because central splitting of the spinous process and lamina is somewhat difficult to perform and technically demanding, meticulous attention must be paid in patients with tiny spinous process and/or patients in whom a large OPLL severely elevates the spinal cord posteriorly to beneath the inner surface of the lamina.

\section{Competing Interests}

The author declares that he has no competing interests.

\section{Acknowledgement}

The author heartily appreciates Mr. Tomohiko Nakano for drawing practical and beautiful figures, and Mr. Larry Frumson for proofreading the manuscript. 
Citation: Hirabayashi S (2017) Surgical Technique and Results of Double-door Laminoplasty at the Cervical Spine (Kurokawa's Method)-Focusing on the Change of Sagittal Alignment. Int J Surg Surgical Porced 2: 118. doi: https://doi.org/10.15344/2456-4443/2017/118

Page 3 of 5

\section{References}

1. Oyama M, Hattori S, Moriwaki N (1973) Trial of one method of cervical laminectomy. Chubu-Seisaishi (Central Japanese Orthopaedic Surgery and Traumatology) 16: 792-794.

2. Kurokawa T, Tsuyama N, Tanaka H, et al. (1982) Double-door laminoplasty by longitudinal splitting of spinous process. Bessatsu Seikeigeka (Suppl Orthop Surg) 2: 234-240.

3. Aita I, Hayashi K, Wadano Y, Yabuki T (1998) Posterior movement and enlargement of the spinal cord after cervical laminoplasty. J Bone Joint Surg $\mathrm{Br}$ 80: 33-7.

4. Shiraishi T, Yato Y, Yoshida H, et al. (2002) New double-door laminoplasty procedures to preserve the muscular attachments to the spinous processes including the axis. Eur J Orthop Surg 12: 175-180.

5. Hirabayashi K, Watanabe K, Wakano K, et al. (1983) Expansive open-door laminoplasty for cervical spinal stenotic myelopathy. Spine (Phila Pa 1976) 8: 693-699.

6. Ito T, Tsuji H (1985) Technical improvements and results of laminoplasty for compressive myelopathy in the cervical spine. Spine 10:729-736.

7. Tsuzuki N, Abe R, Saiki K, lizuka T (1996) Tension-band laminoplasty of the cervical spine. Int Orthop (SICOT) 20: 275-284.

8. Kurokawa T, Tsuyama N, Tanaka H, et al. (1986) Double-door laminoplasty by longitudinal splitting of spinous process-modified procedure and its surgical result. Bessatsu Seikeigeka (Suppl Orthop Surg) 9: 30-32.

9. Hirabayashi S, Kumano K (1995) Development of a new spacer well adapted to split spinous process in double-door laminoplasty. Seikeigeka (Orthop Surg) 46: 110-112.

10. Hoshino Y, Kurokawa T, Hirabayashi S (1987) Double-door laminoplasty by longitudinal splitting of spinous process. Shujutsu (Surgery) 41: 4-10.

11. Asano T, Hirabayashi S (2006) Chapter 133,Surgical management of ossification of the posterior longitudinal ligament. Operative Neurosurgical Techniques Indications, Methods, and Results, Fifth edition, Schmidek HH and Roberts DW ed., Saunders,USA, pp.1879-1895.

12. Hirabayashi S, Kitagawa T, Matsushita T (2013) The devised surgical methods and long-term results of double-door laminoplasty (Kurokawa's method). Sekitsui Sekizui (Spine and Spinal Cord) 26: 1041-1045.

13. Hirabayashi S (2014) Double-door laminoplasty of the cervical spine (Kurokawa's method). Illustrated surgery II-7, Sekitsui Sekizui (Spine and Spinal Cord) 27: 577-581.

14. Hirabayashi S, Kumano K (1999) Contact of hydroxyapatite spacer with split spinous process in double-door laminoplasty for cervical myelopathy. J Orthop Sci. 4:264-268.

15. Hirabayashi S, Kumano K (2000) Finite element analysis of the space created by split spinous processes in double-door laminoplasty to optimize shape of an artificial spacer. Journal of Muscloskeletal Research 4: 47-54.

16. Hirabayashi S, Koshizuka $Y$ (1999) New method of measuring area of spinal canal after double-door laminoplasty. J Orthop Sci. 4:78-82.

17. Hirabayashi S, Murata K, Yamamoto I, et al. (2011) Significance of the maintenance of the spinous process and the stability of an artificial spacer in double-door laminoplasty- Figure analyses. Journal of Spine Research 2: 66-72.

18. Hirabayashi S, Yamada H, Motosuneya T, et al. (2010) Comparison of enlargement of the spinal canal after cervical laminoplasty--- opendoor type and double-door type. Eur Spine J 19: 1690-1694.

19. Hirabayashi S, Matsushita T (2011) Two types of laminoplasty for cervical spondylotic myelopathy at multiple levels. ISRN Orthop 2011: 637185.

20. Fujimura $Y$, Nishi $Y$ (1996) Atrophy of the nuchal muscle and change in cervical curvature after expansive open-door laminoplasty. Arch Orthop Trauma Surg 115:203-205.

21. Sakaura $\mathrm{H}$, Hosono N, Mukai $Y$, et al. (2003) C5 palsy after decompression surgery for cervical myelopathy: review of literature. Spine 28: 2447-2451.
22. Tsuzuki N, Zhogshi L, Abe R, Okai K (1993) Paralysis of the arm after posterior decompression of the cervical spinal cord. 1. Anatomical investigation of the mechanism of paralysis. Eur Spine J 2: 191-196.

23. Hoshino Y, Kurokawa T, Machida H, et al. (1998) Postoperative development of OPLL after double door laminoplasty by longitudina splitting of spinous process. Rinsho Seikeigeka (Clinical Orthopaedics) 23: 531-536.

24. Seich A, Takeshita K, Onishi I, et al. (2001) Long-term results of doubledoor laminoplasty for cervical stenotic myelopathy. Spine 26: 479-487.

25. Hirabayashi S, Saiki K, Sakai H (2004) Occipitocervicothoracic fixation using a rod and hook system for patients with rheumatoid cervical spine. Modern Rheumatology 14: 435-441.

26. Zhang J, Hirabayashi S, Saiki K, Sakai H (2006) Effectiveness of multiplelevel decompression in laminoplasty and simultaneous $\mathrm{C} 1$ laminectomy for patients with cervical myelopathy. Eur Spine J. 15:1367-1374. 